\title{
Les cobénéfices des politiques climatiques: un concept opérant pour les négociations climat ?
}

\author{
Christophe Cassen ${ }^{1}$, Céline Guivarch ${ }^{2}$, Franck Lecocq ${ }^{3}$ \\ 1 Socioéconomiste, CIRED, 94736 Nogent-sur-Marne cedex, France \\ 2 Économiste, CIRED, École des Ponts et Chaussées, 77455 Marne-la-Vallée cedex 2, France \\ 3 Économiste, CIRED, AgroParistech, 75005 Paris, France
}

\begin{abstract}
Mots-clés : environnement ; changement climatique ; cobénéfices ; modélisation intégrée ; analyse coûts-bénéfices
\end{abstract}

\begin{abstract}
Résumé - Cet article ${ }^{1}$ examine les enjeux sous-jacents à l'évaluation des cobénéfices des politiques climatiques dans le cadre de la mise en œuvre de politiques multi-objectifs, au sein lesquelles des synergies sont recherchées entre la lutte contre le changement climatique et des objectifs de développement (emploi, santé, sortie de la pauvreté, etc.). L'analyse du cinquième rapport du groupe III du GIEC montre en effet un intérêt grandissant dans la littérature pour les évaluations quantifiées des cobénéfices, en particulier à l'aide de modèles numériques intégrés. Néanmoins, l'évaluation quantifiée à une échelle globale des cobénéfices est confrontée à des difficultés d'ordre méthodologique qui expliquent l'écart entre les pratiques des modélisateurs et la vision théorique des économistes qui repose sur l'analyse coûts-bénéfices. L'article revient enfin sur la nécessité d'élargir le champ d'investigation de l'évaluation des cobénéfices aux politiques non climatiques qui déterminent une part importante des émissions de GES, et constituent un des enjeux majeurs des approches intégrées climat-développement qui montent en puissance dans les négociations climat en cours.
\end{abstract}

\section{Keywords:}

environment, climate change, co-benefits, climate policies, integrated models, cost-benefits

\begin{abstract}
Co-benefits of climate policies: a potential keystone of climate negotiations? This paper analyzes the challenges related to the assessment of co-benefits of climate policies underpinned by the implementation of multi-objective policies which seek synergies between climate policies and other development objectives (poverty alleviation, employment, health etc.). The analysis highlights the increasing interest in co-benefits in the latest 5th IPCC report, in particular by integrated models. Nevertheless, the quantified evaluation of co-benefits is still confronted to several methodological limitations which reduce the scope of co-benefits, particularly at the global level. In a growing context of climate-development approaches in climate negotiations, this article insists on the need to also assess cobenefits of other policies which induce a significant part of GHG emissions. Considering climate policies focused only on Greehouse Gases emissions reduction limits the range of policy instruments to carbon taxation, tradable carbon emissions permits or dedicated mitigation and adaptation funds. This also hinders the integration of climate objectives in non-climate policies. Analyzing impacts of development policies on Green Gases emissions in the form of co-benefits requires to broaden the range of policy instruments and to take into account other drivers of emissions such as land dynamics. Including these mechanisms in integrated models therefore represents new scientific frontiers for integrated models in the coming years.
\end{abstract}

\footnotetext{
Auteur correspondant : C. Cassen, cassen@centre-cired.fr

1 Ce travail a reçu le soutien de la chaire Modélisation prospective au service du développement durable pilotée par l'École des Ponts Paristech, Mines Paristech, AgroParistech et financée par Edf, l'Ademe, GRTgaz et Schneider Electric.
} 


\section{Introduction}

À quelques mois de la prochaine conférence sur le climat de Paris ${ }^{2}$ et alors que les risques d'aggravation du changement climatique en cas $\mathrm{d}^{\prime}$ inaction se renforcent (IPCC, 2013 ; World Bank, 2014), les négociations internationales ont entamé une dernière ligne droite, avec en ligne de mire l'espoir d'aboutir à un accord global sur le climat qui prendrait la suite du protocole de Kyoto ${ }^{3}$. Cependant, aussi bien dans les pays du Nord que dans ceux du Sud, la préservation du climat doit composer avec d'autres considérations d'ordres économique, politique et social qui ne sont pas sans influencer leur stratégie au sein des négociations. Celles-ci peuvent même constituer un facteur de blocage lorsque l'adoption d'objectifs de réduction des émissions de gaz à effet de serre (GES) est considérée comme un frein potentiel au développement futur (ce qui est le cas dans un certain nombre de pays du Sud et de l'OPEP). Les politiques climatiques ont en effet des conséquences, en plus de la seule lutte contre l'effet de serre, que la littérature qualifie de cobénéfices ou a contrario d'« effets adverses induits », même si comme nous le verrons par la suite la terminologie n'est pas complètement stabilisée. L'évaluation de ces cobénéfices paraît d'autant plus nécessaire que les approches ascendantes qui articulent les politiques climatiques fondées sur des objectifs de réduction des émissions de GES et des enjeux spécifiques de développement ont pris ces dernières années de l'importance dans les négociations climat (Gupta, 2014; Aykut et Dahan, 2015).

Toutefois, la pluralité des conséquences indirectes des politiques climatiques soulève un certain nombre de questions et d'enjeux méthodologiques en matière d'évaluation. Cet article propose ainsi une réflexion sur la notion de cobénéfices, plus précisément au sein de l'expertise produite par la communauté de modélisation intégrée dans le cadre du cinquième rapport d'évalua-

\footnotetext{
2 Les parties à la Convention sur les changements climatiques adoptée à la conférence de Rio en 1992 et entrée en vigueur en 1993 se réunissent chaque année dans le cadre de conférences des parties $(\mathrm{CdP})$. La dernière en date s'est tenue à Lima en décembre 2014

3 La conférence de Durban a mis en place une plateforme en 2011 qui doit servir de base de discussion à l'élaboration d'un accord global en 2015 (UNFCCC Decision 1/CP.17, Dec. 11, 2011, UN Doc. FCCC/CP/2011/9/Add). Dans le cadre du protocole de Kyoto signé en 1997, seuls les pays industrialisés du Nord listés à l'annexe $\mathrm{B}$ du Protocole s'engagent à réduire leurs émissions de gaz à effet de serre de $5 \%$ par rapport à 1990 entre 2008 et 2012. À la conférence de Doha en 2012, le Protocole est prolongé de justesse jusqu'en 2020, mais plusieurs pays dont le Canada, le Japon, la Nouvelle-Zélande et la Russie s'en sont désolidarisés. Le Protocole ne couvre actuellement plus que $15 \%$ des émissions de GES totales.
}

tion du groupe III dédié aux politiques de réduction des émissions de GES ${ }^{4}$. Le chapitre 6 consacré à l'évaluation des «transformation pathways » (Clarke et al., 2014) et les chapitres sectoriels suivant ont analysé 1184 nouveaux scénarios socioéconomiques publiés depuis le quatrième rapport d'évaluation datant de $2007^{5}$. Les scénarios socioéconomiques à long terme évalués ont été produits principalement à partir d'une trentaine de modèles intégrés qui projettent à grande échelle les caractéristiquesclés des «transformation pathways » d'ici 2050 et au-delà. Les modèles intégrés sont des approches simplifiées, stylisées et numérisées, qui représentent en effet les systèmes physiques et sociaux complexes et les interactions les plus pertinentes entre elles (par exemple, l'énergie, l'agriculture, le système économique). Ils utilisent un ensemble d'hypothèses d'entrée et produisent des résultats en sortie tels que les évolutions du système énergétique, de l'usage des sols, les effets économiques des mesures d'atténuation, et les trajectoires d'émissions (Sarofim et Reilly, 2011). Ces modèles, en représentant les effets systémiques entre différents domaines, ont par conséquent la possibilité de pouvoir évaluer les effets directs et indirects des politiques climatiques, en particulier à l'échelle globale (Encadré).

La première partie de cet article revient sur la progressive stabilisation d'une notion au périmètre large et sur les enjeux qu'elle présente pour les économistes. La deuxième partie analyse la montée en puissance des évaluations des cobénéfices par la modélisation intégrée, en particulier depuis le quatrième rapport du Groupe d'experts intergouvernemental sur l'évolution du climat (GIEC) publié en 2007 et les principaux résultats tirés de la littérature. La troisième partie se concentre sur les limites méthodologiques rencontrées par les modèles intégrés pour évaluer les cobénéfices. Enfin, dans une quatrième partie, nous verrons comment l'articulation entre ces évaluations et les dynamiques récentes des négociations climat, marquées par l'émergence d'approches multi-objectifs dans lesquelles enjeux climatiques et de développement sont associés, nécessitent d'approfondir la représentation dans les modèles des mécanismes à l'origine des cobénéfices.

\footnotetext{
4 Il ne s'agit pas d'une analyse textuelle de la place des cobénéfices dans la littérature centrée sur les négociations climat, ni d'une histoire des politiques multi-objectifs.

5 À titre de comparaison, le quatrième rapport évaluait 780 scénarios ; le troisième, 380 scénarios avec 26 modèles et le deuxième, seulement 6 scénarios.
} 


\section{Encadré. La communauté des modèles intégrés}

La communauté des modèles intégrés est composée d'environ une trentaine de modèles dans le monde. Ces modèles se sont développés au sein d'instituts de recherche (à l'origine principalement dans les pays de l'OCDE avant de s'étendre dans les pays du Sud : Inde, Chine, Afrique du Sud et Brésil notamment), de grandes organisations internationales (OCDE et Agence internationale de l'énergie) ou de structures gouvernementales (comme le PBL/Netherlands Environmental Agency ou le ministère australien de l'Agriculture). Au sein des pays de l'OCDE, les principaux instituts de recherche sont situés aux États-Unis : Pacific Northwest Laboratory, Massachusetts Institute of Technology, Stanford ; en Europe : Potsdam Institute for Climate, University College of London, Fondazione Eni Enrico Mattei (FEEM), PBL, International Institute for Applied Systems Analysis (IIASA), Centre international de recherche sur l'environnement et le développement (CIRED), Edden/Université de Grenoble, Centre de mathématiques appliquées (CMA) principalement ; en Australie et au Japon : National Institute for Environmental Studies. La communauté des modèles intégrés s'est structurée dans le cadre de réseaux de recherche comme 1'Energy Modeling Forum (EMF), l'IAMC (Integrated Assessment Modeling Consortium) à Stanford ou l'ETSAP (Energy Technology System Analysis Program), de centres de recherches tel l'IIASA à Vienne, et d'exercices de comparaison de modèles, en particulier financés dans le cadre du septième programme-cadre européen qui ont fourni une grande partie des scénarios socioéconomiques analysés dans le cinquième rapport du GIEC. Les interactions avec d'autres communautés (spécialistes des enjeux relatifs à la biodiversité, économistes mainstream, sciences sociales ou encore sciences de la terre) ne sont pas vraiment institutionnalisées, mais plutôt le fait de collaborations informelles. Une initiative comme la plateforme internationale de recherche Future Earth, fondée en 2012 à la suite de la conférence Rio+20 et destinée à encourager et financer des programmes de recherche interdisciplinaires en vue d'alimenter le processus de décision, pourrait accélérer ces rapprochements.

\section{Une notion en quête d'une définition stabilisée}

\section{Les contours flous des cobénéfices}

Les cobénéfices des politiques climatiques couvrent un périmètre très large et multidimensionnel en matière de santé, d'impact sur l'environnement, d'accès à l'énergie, d'emplois, etc. Par exemple, le remplacement de centrales électriques au charbon par des centrales au gaz ou alimentées par des énergies renouvelables, l'introduction de mesures en faveur de la diminution du trafic routier ou de l'amélioration du confort thermique des bâtiments peuvent générer des impacts positifs sur la santé (en limitant les émissions de GES et de particules fines), la sécurité énergétique (en baissant la facture des ménages), et la préservation de l'environnement. Cette diversité n'est sans doute pas étrangère au flou sémantique qui entoure la notion de cobénéfices, comme le suggère la liste des acceptions répertoriées dans la littérature par Ürge-Vorsatz et al. (2014). Si l'on s'en tient aux travaux du groupe III du GIEC, l'existence des cobénéfices des politiques climatiques est mentionnée dès le deuxième rapport (IPCC, 1996) mais le terme s'est imposé tardivement, après une série de glissements sémantiques ${ }^{6}$. Dans le deuxième rapport, on ne parle pas encore de cobénéfices, mais

\footnotetext{
6 Les premières évocations des cobénéfices figurent dans une conférence organisée conjointement par l'UNEP, WMO et ICSU en 1985 sur le changement climatique ainsi que dans la contribution de Crutzen et Graedel à l'ouvrage Sustainable Development and the Biosphere (1987).
}

de bénéfices involontaires indirects (secondary benefits) ou environnementaux connexes (ancillary environmental effects) des politiques d'atténuation des émissions de GES. En 2000, 1'OCDE organise un colloque sur le sujet dont les principales conclusions seront reprises dans le troisième rapport du GIEC. On cherche à cette époque à déterminer si la recherche des cobénéfices constitue un objectif explicite des politiques climatiques. C'est à la suite de ce colloque que le terme de "cobénéfice » est introduit, mais contrairement aux effets connexes présentés dans le deuxième rapport, il constitue un objectif explicite des politiques climatiques globales ou sectorielles (en matière d'efficacité énergétique, d'emploi, de transport, etc.). Les cobénéfices résultent spécifiquement de politiques qui intègrent les enjeux climatiques et de développement (la réduction des émissions reste toutefois l'objectif premier). Cette vision est reprise dans le cinquième rapport (IPCC, 2014) qui distingue les cobénéfices des effets adverses induits, même si les termes «bénéfices connexes » ou bénéfices indirects (entre autres) restent encore utilisées dans une partie de la littérature.

\section{Les cobénéfices vus par les économistes}

Du point de vue des économistes qui constituent la communauté prédominante dans le groupe III, en corrigeant certaines externalités négatives dont la source est liée aux émissions de gaz à effet de serre (dans le cas de la pollution urbaine par exemple), ou des imperfections de marché (sur le marché de l'emploi en particulier), la mise en œuvre de politiques climatiques peut être à l'origine 
de cobénéfices. L'analyse des cobénéfices n'est pas en soi nouvelle en économie, puisqu'elle renoue avec une discussion théorique qui a trait à la possibilité qu'une politique climatique s'accompagne d'un double dividende (baisse des émissions de GES et gain économique associé). Au cours des années 1990, une partie de la littérature macroéconomique s'est en effet polarisée sur les effets engendrés par une substitution de certaines taxes existantes, en particulier sur le travail, en introduisant une fiscalité carbone. Certaines études ont montré qu'une réforme fiscale de ce type pouvait s'accompagner d'un gain macroéconomique net que l'on qualifie de double dividende au sens "fort", même si ce résultat demeure encore aujourd'hui controversé ${ }^{7}$.

Une autre caractéristique du point de vue économique des cobénéfices est que, contrairement au climat, ils ne constituent pas un bien public global ${ }^{8}$, en raison de leur horizon spatiotemporel. En effet, les bénéfices directs apportés par la réduction des gaz à effet de serre sont avant tout globaux alors que les cobénéfices, comme la réduction de la pollution de l'air, sont directement observables à l'échelle locale ou régionale (IPCC, 1996 ; Pearce, 1992). Les cobénéfices sont par ailleurs exploitables à court terme, alors que les bénéfices directs des réductions des émissions peuvent prendre des décennies avant d'être visibles. Ces traits propres aux cobénéfices ne sont pas sans répercussions méthodologiques, en particulier lorsqu'il s'agit de conduire des analyses coûtsbénéfices comme nous le verrons par la suite.

Après avoir clarifié ces enjeux de définition des cobénéfices, nous allons voir à présent comment, en marge des analyses portant sur l'évaluation du coût des politiques climatiques, les cobénéfices ont progressivement constitué un objet de recherche en soi. Le cinquième rapport du GIEC a approfondi à la fois le cadrage conceptuel et celui en matière d'évaluation, à la suite des premières tentatives au début des années 2000.

\section{La montée en puissance des cobénéfices dans le cinquième rapport du GIEC}

\section{Une place grandissante dans le rapport du groupe III du GIEC}

L'existence de potentiels cobénéfices des politiques climatiques est mentionnée dès le milieu des années 1990 dans

\footnotetext{
7 La définition de la forme « forte » du double dividende est donnée par Goulder (1995) : «It is possible to find a distortionary tax such that the revenue-neutral substitution of the environmental tax for this tax involves a zero or negative gross cost » (p. 4). On parle de double dividende " faible » lorsque cette amélioration ne compense que partiellement les coûts (Goulder, 1995). Pour un tour d'horizon récent sur ce débat voir Combet (2013).

8 Un bien public global est un bien que l'on qualifie de non rival et de non exclusif.
}

les travaux du GIEC, mais leur analyse reste secondaire par rapport à l'évaluation des coûts des politiques climatiques. En effet, la littérature s'est concentrée principalement sur l'évaluation quantifiée du coût pour l'économie d'objectifs de réduction des émissions, à l'aide de modèles numériques intégrés ou macroéconomiques, en particulier à la suite de l'adoption du protocole de Kyoto en 1997 et de l'institutionnalisation progressive de l'objectif $2^{\circ} \mathrm{C}^{9}$.

Le cinquième rapport du groupe III constitue un saut quantitatif et qualitatif dans l'analyse des cobénéfices des politiques climatiques. Le nombre d'occurrences du terme cobénéfices augmente (61 dans le résumé technique du cinquième rapport ${ }^{10}$ vs 31 occurrences dans le quatrième). Les évaluations sont plus systématiques au niveau sectoriel et synthétisées sous la forme d'un tableau dans les chapitres 8 à 12 consacrés respectivement au transport, au résidentiel, à l'industrie, à l'agriculture et à la ville. Le tableau ci-après présente par exemple les cobénéfices économiques, environnementaux et sociaux des politiques d'atténuation à l'échelle urbaine. Chaque tableau sectoriel s'appuie sur une littérature croissante sur le sujet (215 références pour le chapitre 6 consacrées aux enjeux énergétiques), avec un traitement plus ou moins explicite des cobénéfices, à différentes échelles d'analyse (globale, locale, sectorielle), et suivant différentes méthodes (évaluations plus ou moins quantifiées). Comme il n'est pas possible de résumer l'ensemble de ces travaux dans le cadre de cet article, on se contentera d'en dégager les principaux traits.

\section{Un recours limité mais croissant aux modèles intégrés}

Deux types d'approches, qualitatives ou quantifiées, sont utilisés dans la littérature répertoriée par le GIEC pour évaluer les cobénéfices et les effets adverses induits des politiques climatiques. Les approches quantifiées mobilisent des modèles de nature variée en fonction des domaines analysés (santé, pollution, etc.). Cet article, s'intéresse uniquement aux résultats quantifiés récents d'un type particulier de modélisation dit intégré qui regroupe une grande partie des modèles du chapitre 6 du groupe III du GIEC ${ }^{11}$.

\footnotetext{
9 L'objectif de limiter l'augmentation de la température mondiale globale à $2{ }^{\circ} \mathrm{C}$ a été formellement intégré dans les négociations climat lors de la conférence de Cancún en 2010 (http:/ / unfccc.int/files/meetings/cop_16/application/pdf/ cop16_lca.pdf).

${ }^{10}$ Les rapports des trois groupes du GIEC comportent chacun un rapport final, un résumé technique et un résumé pour les décideurs.

11 Ces modèles peuvent toutefois être couplés à des modules plus spécifiques pour évaluer les impacts des politiques de réduction des gaz à effet de serre sur la santé, la biodiversité, les émissions de particules fines.
} 
Tableau. Potentiels cobénéfices (flèches en trait plein) et risques d'effets adverses induits (flèches en pointillés) des principales mesures d'atténuation à l'échelle urbaine.

\begin{tabular}{|l|l|l|l|}
\hline \multirow{2}{*}{$\begin{array}{c}\text { Mesures d'atténuation du } \\
\text { changement climatique }\end{array}$} & \multicolumn{1}{|c|}{ Effets sur d'autres enjeux } \\
\cline { 2 - 4 } & \multicolumn{1}{|c|}{ Économiques } & \multicolumn{1}{|c|}{ Sociaux (y compris santé) } & \multicolumn{1}{c|}{ Environnementaux } \\
\hline $\begin{array}{l}\text { Développement urbain et } \\
\text { infrastructures denses }\end{array}$ & $\begin{array}{l}\uparrow \text { Innovation et productivité } \\
\uparrow \uparrow \text { Loyers et valeur du fon- } \\
\text { cier plus élevés } \\
\uparrow \text { Distribution et usage des } \\
\text { ressources efficaces }\end{array}$ & $\begin{array}{l}\uparrow \text { Santé (activité physique) } \\
\text { naturels }\end{array}$ & \\
\hline Amélioration de l'accessibilité & $\begin{array}{l}\uparrow \text { Réduction des coûts dus aux } \\
\text { trajets domicile-travail }\end{array}$ & $\begin{array}{l}\uparrow \text { Santé (activité physique) } \\
\text { Interactions sociales et bien- } \\
\text { etre mental }\end{array}$ & $\begin{array}{l}\uparrow \text { Qualité de l'air et impacts } \\
\text { surcosystèmes et la santé }\end{array}$ \\
\hline $\begin{array}{l}\text { Aménagement foncier mixte } \\
\text { (résidentiel, emplois, com- } \\
\text { merces) }\end{array}$ & $\begin{array}{l}\uparrow \text { Réduction des coûts (finan- } \\
\text { ciers et temps) des trajets } \\
\text { domicile-travail } \\
\uparrow \text { 个 Loyers et valeur du } \\
\text { foncier plus élevés }\end{array}$ & $\begin{array}{l}\uparrow \text { Santé (activité physique) } \\
\text { Interactions sociales et } \\
\text { bien-être mental }\end{array}$ & $\begin{array}{l}\uparrow \text { Qualité de l'air et impacts } \\
\text { sur les écosystèmes et la santé }\end{array}$ \\
\hline
\end{tabular}

Les travaux synthétisés dans les chapitres sectoriels du cinquième rapport du GIEC (groupe III) couvrent de manière inégale l'ensemble du spectre des cobénéfices. Toute une série d'études s'intéresse aux cobénéfices en matière de santé liés à la réduction de la pollution de l'air (baisse de la mortalité et des maladies respiratoires engendrées par la pollution) alors que d'autres thèmes comme les impacts en matière de biodiversité ont été moins explorés jusqu'à présent. Plusieurs raisons expliquent cet intérêt variable comme la disponibilité inégale des données en fonction des domaines ou encore l'intérêt croissant pour les questions liées à la pollution de l'air depuis les années 1980 dans les pays du Nord puis du Sud. Plus récemment, l'analyse quantifiée des cobénéfices en matière de sécurité énergétique a pris de l'ampleur, principalement sous l'impulsion de l'étude intitulée Global Energy Assessment (GEA) ${ }^{12}$ et dans un contexte de tensions croissantes sur les marchés de l'énergie.

Par ailleurs, peu d'études ont recours à des modèles intégrés. Seulement une dizaine dans les années 1990 utilisent des modèles, plutôt technicoéconomiques ou «bottom up (ascendants) qui ont l'avantage de représenter finement un ou plusieurs secteurs, mais beaucoup moins bien les relations avec le reste de l'économie (Markandya et Rübbelke, 2004 ; Pearce, 2000). Néanmoins, depuis la publication du quatrième rapport du GIEC en 2007, on note un regain d'intérêt pour les

\footnotetext{
12 Cette vaste étude a mobilisé quelque 300 experts couvrant un large champ de disciplines. Coordonnée par l'IIASA, elle analyse les transformations nécessaires à l'échelle globale et sectorielle des systèmes énergétiques afin de rendre compatibles les objectifs de réduction des émissions de GES, de sécurité énergétique et d'accès à l'énergie pour tous.
}

cobénéfices des politiques climatiques dans la communauté des modélisateurs intégrés. Parmi les travaux les plus notables, nous avons déjà mentionné le GEA, étude publiée en 2012, dont une partie des résultats a fait l'objet de publications dans des revues de premier rang comme Climatic Change et Nature Climate Change (Mc Collum et al., 2011 ; Mc Collum et al., 2013). Le GEA évalue plus précisément la compatibilité entre la mise en place de politiques d'atténuation des émissions ambitieuses (objectif $2{ }^{\circ} \mathrm{C}$ ), de sécurité énergétique et de limitation de la pollution locale. En parallèle, un numéro spécial paraît dans The Lancet en 2009 sur les bénéfices en matière de santé des politiques climatiques dans différents domaines (agriculture, bâtiment, transport, production d'électricité). Une partie des articles publiés dans ce numéro spécial utilise des modèles intégrés couplés avec des modules d'évaluation de la pollution et de santé dont les résultats sont abondamment cités dans le cinquième rapport du GIEC $^{13}$.

Les évaluations des cobénéfices répertoriées dans le rapport du GIEC privilégient en général l'échelle locale ou sectorielle comme l'indique la légende du tableau présenté précédemment. Les modèles intégrés, au contraire, apportent une vision plus globale ou à

\footnotetext{
${ }^{13}$ En particulier dans le cadre de l'article de Markandya et al. (2009) consacré aux cobénéfices liés à la production d'électricité peu carbonée dans le domaine de la santé. Le modèle POLES y projette des modes de production d'électricité en fonction des objectifs de réduction des émissions de $\mathrm{CO}_{2}$, alors que le modèle GAINS évalue la concentration des particules fines d'un diamètre de $2.5 \mu \mathrm{m}\left(\mathrm{PM}_{2.5}\right)$. Un autre modèle quantifie les effets des $\mathrm{PM}_{2.5}$ sur la mortalité en ayant recours à des méthodes d'évaluation comparée des risques, mises au point par l'Organisation mondiale de la santé.
} 
l'échelle de grandes régions. Les études ayant recours à ces modèles se sont concentrées à l'origine plutôt sur l'Europe et les États-Unis, avant de s'étendre progressivement aux pays en développement. ${ }^{14}$ Les problèmes de santé liés à l'augmentation du trafic routier dans les mégalopoles des pays émergents ou encore à l'utilisation de la biomasse comme bois de chauffage ont en effet augmenté significativement depuis une vingtaine d'années, au point de devenir une priorité pour les gouvernements (IPCC, 2014). La section suivante présente les principaux résultats de la littérature synthétisés dans le rapport du groupe III du GIEC.

\section{Globalement les cobénéfices des politiques climatiques l'emportent sur les effets négatifs}

Le cinquième rapport du GIEC souligne l'existence de cobénéfices substantiels des politiques climatiques. Dans le domaine de la pollution de l'air et de la santé, les travaux publiés depuis 2007 confirment que les efforts d'atténuation compatibles avec l'objectif $2{ }^{\circ} \mathrm{C}$ sont susceptibles de produire d'importants cobénéfices. Riahi et al. (2012) dans le GEA évaluent à l'aide du modèle MESSAGE $^{15}$ à respectivement $50 \%, 35 \%$ et $30 \%$ d'ici 2030 les baisses d'émissions de dioxyde de soufre $\left(\mathrm{SO}_{2}\right)$, de dioxyde d'azote (NOx) et de particules fines (PM2.5), tandis que West et al. (2013) concluent à $0,5 \pm 0,2$ et $1,3 \pm 0,5$ millions de morts prématurés en moins en 2030 et 2050, à l'échelle globale. Les pays en développement sont particulièrement concernés par ces potentiels cobénéfices, car ils disposent en général de législations antipollution moins strictes que dans les pays développés ${ }^{16}$. A contrario, ces cobénéfices sont en partie contrebalancés par certains effets négatifs des politiques d'atténuation. L'augmentation des prix des énergies conventionnelles induite par une contrainte carbone peut en effet favoriser, à court terme, l'emploi d'énergies

\footnotetext{
14 Parmi les études marquantes dans les années 1990, il convient de noter celles de Glomsrod et al. (1992) sur la pollution de l'air dans les pays scandinaves ou encore celle de Barker et al. (1993) qui évalue à l'aide d'un modèle économétrique les effets d'une taxe carbone/énergie sur le trafic routier en GrandeBretagne. À la suite de l'étude pionnière de Complainville et Martins (1994), plusieurs travaux modélisés se sont intéressés dans les années 2000 aux grands pays émergents comme la Chine et l'Inde. Parmi ceux-ci, Bussolo et O'Connor (2001) pour l'Inde, Garbaccio et al. (1999) puis O'Connor et al. (2003) pour la Chine.

${ }^{15}$ L'architecture de modélisation intégrée MESSAGE (Model for Energy Supply Strategy Alternatives and their General Environmental Impact) est composée d'un modèle d'optimisation technicoéconomique du système énergétique couplé avec le modèle climatique MAGICC (Model for the Assessment of Greenhouse-gas Induced Climate Change).

${ }^{16}$ Les fortes densités urbaines en Inde et en Chine constituent également un facteur important.
}

non commerciales à base de biomasse pour le chauffage et la cuisine qui s'accompagne d'une augmentation de la pollution de l'air intérieur avec des impacts sur la santé.

Dans les scénarios où des politiques de réduction des émissions ambitieuses compatibles avec l'objectif $2^{\circ} \mathrm{C}$ sont mises en œuvre, la sécurité énergétique des pays développés et en développement s'améliore également. Toujours avec le modèle MESSAGE, le GEA évalue les effets positifs à la fois sur la souveraineté énergétique des pays importateurs, la résilience et la robustesse de leur système énergétique ${ }^{17}$. Les politiques climatiques contribuent en effet à réduire la demande d'énergies fossiles et améliorent l'intensité énergétique de l'économie. Elles limitent ainsi la dépendance énergétique par une baisse des importations d'énergie fossile, tout en favorisant la diversification de la production énergétique, notamment vers les énergies renouvelables. Ces effets positifs peuvent néanmoins s'accompagner, entre autres, d'une augmentation de la précarité énergétique des ménages les plus pauvres liée à la hausse des prix de l'énergie (principalement les prix de l'électricité), ou comme le montrent d'autres études, d'une baisse de la rente pétrolière des pays exportateurs de pétrole (Waisman et al., 2013). Enfin, le GEA souligne que les objectifs de réduction des émissions peuvent être atteints plus efficacement si les objectifs climatiques et de développement sont poursuivis simultanément plutôt que de manière isolée (McCollum et al., 2011 ; 2013 ; Riahi et al., 2012). Les économies réalisées sur les dépenses liées au contrôle de la pollution et à la sécurité énergétique grâce à la mise en synergie de ces politiques sont potentiellement significatives, estimées entre 100 et 600 milliards de \$ US par an d'ici 2030 (0,1-0,7\% du PIB) [McCollum et al., 2013].

Cet aperçu synthétique de la littérature reflète le relatif optimisme des modèles en matière de cobénéfices des politiques climatiques, en particulier dans le cadre de politiques intégrées ou multi-objectifs. Ces résultats ne doivent toutefois pas masquer les enjeux auxquels l'analyse coûts-bénéfices est confrontée pour évaluer les cobénéfices comme nous allons le voir à présent.

\section{Les évaluations coûts-bénéfices au prisme des cobénéfices}

\section{Des méthodologies diverses}

Les évaluations des cobénéfices par les modèles intégrés se présentent principalement sous deux formes, physique ou monétaire. Les analyses "physiques » évaluent les cobénéfices de manière purement quantitative : par exemple, l'impact sur l'espérance ou la qualité de vie

\footnotetext{
17 Ces résultats corroborent des études antérieures citées dans le quatrième rapport du GIEC.
} 
lié à l'amélioration de la qualité de l'air ou l'accroissement du confort thermique des bâtiments (en fonction de la température intérieure ou de la superficie chauffée en $\mathrm{m}^{2}$ ) qui résulte de mesures en faveur de l'efficacité énergétique.

Les évaluations « monétaires » des cobénéfices consistent à rechercher le «coût total net » de la politique en intégrant dans une métrique commune le coût direct, les cobénéfices et les effets adverses induits qui relèvent principalement de l'analyse coûts-bénéfices. Le chapitre $3 \mathrm{du}$ cinquième rapport du groupe III consacré aux aspects méthodologiques et conceptuels de l'évaluation économique des politiques climatiques propose un cadre d'analyse formalisé d'évaluation qui prend en compte tous les bénéfices des politiques climatiques et leur impact sur le bien-être global :

$$
\partial V=\sum_{i=1}^{m} \frac{\partial z_{i}}{\partial p_{1}} \frac{\partial V}{\partial z_{i}} \partial p 1
$$

Équation 1: calcul des variations du bien-être en fonction des cobénéfices (IPCC, 2014)

Le bien-être global dépend des interactions entre une politique climatique et différents objectifs environnementaux, économiques et sociaux poursuivis. Il suppose dans un premier temps d'identifier les objectifs impactés par une politique climatique $(\mathrm{i}=1, \ldots, \mathrm{m})$. La première composante de l'équation $1, \frac{\partial z_{i}}{\partial p_{1}}$, évalue ensuite de manière physique l'impact d'une politique climatique $(\partial p 1>0)$ sur différents objectifs : sous la forme de bénéfices directs - limitation de la montée de la température à l'échelle globale (z1) et du niveau de la mer (z2), maintien de la productivité des sols (z3) dans le cas présent et sous la forme de cobénéfices - réduction des émissions de dioxyde de soufre (z4), amélioration de la sécurité énergétique (z5) ou encore maîtrise de l'étalement urbain (z6). La seconde composante, $\frac{\partial V}{\partial z_{i}}$, évalue monétairement la valeur sociale associée à chaque unité de cobénéfices : par exemple, la valeur accordée à une unité de dioxyde de soufre en moins diffusée dans l'atmosphère. La multiplication et la sommation de ces deux composantes donnent la valeur du bien-être global sous la forme d'un indicateur global $(\partial V)$.

Cette équation constitue un cadre conceptuel « idéal » d'évaluation des cobénéfices directement issu de la théorie économique des cobénéfices. Toutefois, les pratiques d'évaluation des cobénéfices par les modélisateurs intégrés, comme nous allons l'aborder à présent, sont confrontées à de nombreux obstacles méthodologiques qui limitent le recours aux analyses coûtsbénéfices classiques.

\section{Un écart important entre la théorie économique et la pratique des modélisateurs intégrés}

Les avantages et les inconvénients d'évaluer des coûts et des bénéfices nets d'une politique environnementale ont fait l'objet de nombreux débats dans la littérature, notamment sur la disponibilité des prix du marché pour évaluer les biens environnementaux et la difficulté d'agréger des effets locaux (Pearce, 1976; 2001). Dans le domaine du changement climatique, l'évaluation des bénéfices des politiques climatiques est de manière générale confrontée à de nombreuses incertitudes relatives aux évolutions du climat pour un scénario d'émissions données, à la réponse des grands cycles géophysiques (cycle de l'eau par exemple) et des écosystèmes, aux évolutions des modes de vie et de la richesse d'une société, ou encore à la représentation des impacts du changement climatique au sein des modèles ${ }^{18}$.

L'identification et la quantification physique des cobénéfices $\left(\frac{\partial z_{i}}{\partial p_{1}}\right.$ dans l'équation 1$)$ sont empreintes de nombreuses incertitudes, en particulier à grande échelle. Les cobénéfices, comme nous l'avons souligné plus haut, sont très dépendants du contexte local. Les données quant aux impacts des politiques climatiques sur la santé humaine, par exemple, sont issues pour la plupart de monographies locales dont l'extrapolation à un pays entier est relativement complexe ${ }^{19}$. Par ailleurs, l'amplitude des cobénéfices dépend du type de politiques déjà mises en œuvre dans le scénario de référence (baseline). Un scénario avec politiques climatiques comparé à une baseline considérée par un modèle comme optimale (par exemple sans chômage et/ou avec des politiques antipollution) est peu susceptible de s'accompagner de cobénéfices ${ }^{20}$. Enfin, les interrelations entre les différents impacts (les $z_{i}$ de l'équation 1) rendent l'identification de l'ensemble des cobénéfices très complexe, ce qui accroît le risque de double comptabilité.

L'évaluation monétisée de l'impact des cobénéfices sur le bien-être $\left(\frac{\partial V}{\partial z_{i}}\right)$ est également problématique. Le bien-être social est une notion complexe qui fait intervenir différentes dimensions (santé, prospérité et bonheur). Les économistes l'appréhendent au travers des variations de l'utilité mesurées par la variation du surplus ou la variation compensatoire de revenu, ou via des

\footnotetext{
18 Considérer que les impacts suivent une fonction linéaire ou non peut grandement influer sur les résultats (Ambrosi et al.,2003).

${ }^{19}$ Ceci explique sans doute la faiblesse des analyses coûtsbénéfices globales qui tranche avec l'habitude d'appliquer ce genre d'approche à l'échelle de projets d'infrastructures locaux.

${ }^{20}$ La structure des modèles peut expliquer ce genre de choix, en particulier au niveau des modèles d'optimisation.
} 
proxi comme la consommation, et de manière plus indirecte par le PIB. Mais, dans les faits, la plupart des études modélisées se contentent d'évaluer les économies en matière de dépenses (par exemple de santé) engendrées par les cobénéfices, sur le coût des politiques climatiques (exprimé par les variations du PIB) ou sur la valeur du carbone nécessaire à l'atteinte d'un objectif exprimé en $\$ \mathrm{US} / \mathrm{tC}^{21}$. Certains paramètres-clés dans les évaluations monétisées comme la valeur accordée à une vie humaine ont par ailleurs fait l'objet par le passé de vives controverses au sein de la communauté, en particulier au moment de la rédaction du deuxième rapport du GIEC. Les estimations du coût d'une vie humaine, plus élevées dans les pays du Nord en raison de la différence de niveau de richesse, ont pu laisser croire que la vie d'un Africain avait moins de valeur que celle d'un Européen (IPCC, 1996 ; Grubb, 1999) et artificiellement gonflé les cobénéfices dans les pays du Nord ${ }^{22}$. Enfin, un indicateur de bien-être présenté de manière agrégée ne permet pas de distinguer quelles composantes de la société (types de ménages, de secteurs, etc.) sont les plus affectées par les politiques climatiques.

On comprend ainsi mieux pourquoi la plupart des analyses modélisées récentes se contentent d'évaluer le plus souvent les impacts d'un seul cobénéfice (à l'exception notable des travaux du GEA qui s'intéresse aux synergies entre objectifs), en privilégiant la dimension physique.

Les limites rencontrées par l'évaluation des cobénéfices des politiques climatiques invitent par conséquent à se montrer prudents par rapport aux résultats des modèles de prospective intégrés. Elles laissent entrevoir l'étendue des chantiers de recherche, en particulier au regard des évolutions récentes dans les négociations climat comme nous allons le voir à présent.

\section{Une notion susceptible de dénouer le « nœud gordien climat/développement » 23 dans les futures négociations ?}

\section{Une clé de voûte potentielle des politiques multi-objectifs}

La question des cobénéfices a été jusqu'à présent peu prise en compte dans les négociations climat. Celles-ci ont privilégié l'adoption d'objectifs de réduction d'émissions dans le cadre du protocole de Kyoto, et plus récemment, en institutionnalisant l'objectif des $2{ }^{\circ} \mathrm{C}$. Un marché du carbone (Cap and Trade) a constitué le principal instrument

\footnotetext{
${ }^{21}$ Ces analyses se contentent d'une analyse coût/efficacité qui $\mathrm{n}$ 'inclut pas les dommages évités par les politiques climatiques.

22 Bien que la valeur de la vie augmente avec le temps en raison de l'accroissement des richesses et de l'accélération de l'urbanisation, entraînant par là une augmentation des cobénéfices.

23 Cette expression est empruntée à Hourcade et al. (2008).
}

de réduction des émissions mis en œuvre à l'échelle internationale. Cette vision « climato-centrée » des négociations qui place en haut de la hiérarchie des priorités la réduction des émissions de GES avant les enjeux de développement a montré ses limites au cours de la conférence de Copenhague en 2009, face à l'opposition des pays émergents. Ces derniers refusent en effet d'intégrer un système avec des objectifs de réduction contraignants qui limiterait à terme leur croissance, et ce conformément au principe des responsabilités communes mais différenciées inscrit dans la Convention sur les changements climatiques de 1992 (Hourcade et Shukla, 2013).

Une approche plus ascendante des négociations climat a néanmoins émergé ces dernières années qui tente d'intégrer les enjeux climatiques dans les politiques de développement. Chaque pays impliqué dans le processus de négociation peut proposer des politiques de réduction des émissions cohérentes avec ses enjeux de développement propres, au sein des NAMAs (Nationally Appropriate Mitigation Actions) dont le cadre a été établi lors des conférences de Copenhague (2009) et Cancún (2010), et qui s'intègrent dans les "contributions" nationales en faveur de l'atténuation d'ici fin $2015^{24}$ (Bodansky et Diringer, 2014 ; Damian, 2014 ; UNFCCC, 2014). Cette approche rencontre un écho en particulier dans les pays émergents dont certains ont mis en œuvre des programmes nationaux de lutte contre le changement climatique, articulés autour de leurs propres objectifs de développement. C'est le cas notamment du National Action Plan on Climate Change (NAPCC) en Inde (Dubash, 2013 ; Dubash et al., 2013).

En recentrant ainsi les politiques climatiques sur les priorités nationales de développement et leurs potentiels cobénéfices, les efforts d'atténuation pourraient acquérir une plus grande légitimité auprès de populations sensibles aux effets de court terme sur les prix des énergies des politiques climatiques. En effet, la plupart des politiques qui ont un impact sur les réductions des émissions mises en œuvre sont motivées par des raisons qui ne sont pas stricto sensu climatiques, mais liées à d'autres enjeux comme la sécurité énergétique ou la pollution atmosphérique urbaine. Des politiques climatiques phares comme le marché de carbone européen ne doivent pas faire oublier que la lutte contre le changement climatique est de fait considérée dans bien des cas comme un objectif parmi d'autres, voire même comme un cobénéfice des politiques de développement. Les objectifs ambitieux en matière d'efficacité énergétique de la Chine se déploient ainsi sur fond d'une course effrénée aux ressources énergétiques dans le monde (Sovacool et Brown, 2010),

\footnotetext{
${ }^{24}$ Les parties à la Convention sur les changements climatiques sont invitées à soumettre courant 2015 leurs Intended Nationally Determined Contributions (INDCs) en matière de réduction des émissions, mais aussi d'adaptation.
} 
pendant que les États-Unis appliquent des standards plus stricts en matière de consommation de carburants par véhicule (Anderson et al., 2011), afin de réduire d'un tiers leur dépendance pétrolière d'ici 2025 (the White House, 2011). Même à l'échelle d'une ville, les politiques prises en compte dans le tableau présenté précédemment comme la densification urbaine ont bien souvent comme origines principales, entre autres, l'accès problématique au foncier des couches moyennes ou les problèmes de congestion. En structurant l'espace urbain, les politiques d'urbanisme jouent un rôle-clé dans la construction des besoins en transport des ménages et des entreprises, et dans les émissions de GES.

\section{Les enjeux de la représentation des politiques non climatiques dans les modèles intégrés}

L'exemple des politiques urbaines souligne combien les politiques «non climatiques » déterminent une part importante des émissions de GES ainsi que la capacité à s'adapter, et constituent des leviers essentiels pour réaliser des objectifs ambitieux de lutte contre le changement climatique (Hourcade et Shukla, 2013). Des études modélisées de 1'IIMA (Indian Institute of Management Ahmedabad) ou du CIRED avec le modèle Imaclim montrent ainsi que la prise en compte des cobénéfices liés à la mise en place de politiques complémentaires à la fixation d'un prix du carbone, par exemple dans le transport, tend à diminuer le coût net des politiques climatiques, en particulier dans les pays en développement (Shukla et Dhar, 2011 ; Waisman et al., 2012). Ces résultats rejoignent les conclusions du GEA sur l'importance de conduire des politiques multi-objectifs qui rendent compatibles la sortie de la pauvreté pour des centaines de millions de personnes dans les pays du Sud dans les prochaines décennies et la poursuite d'objectifs ambitieux de réduction des émissions.

La mise en cuvre des politiques multi-objectifs mérite également que l'on étende le champ des instruments des politiques climatiques. Une vision de ces politiques centrée uniquement sur les réductions d'émissions tend en effet à privilégier des formes d'action publique très spécifiques comme la fiscalité carbone, des marchés de permis négociables ou des fonds dédiés (à l'atténuation et à l'adaptation), qui ont tendance à occulter tout ce qui relève d'une meilleure intégration de l'enjeu climatique au sein des politiques non climatiques (Lecocq, 2015). En revanche, la prise en compte des impacts sur les émissions des politiques de développement en tant que cobénéfices permet d'élargir le champ des instruments et de s'intéresser à des mécanismes qui influencent les émissions, par exemple ceux relatifs aux dynamiques foncières (fiscalité en particulier) ou encore plus largement au financement de l'économie (voir la contribution de E. Espagne et al. dans ce numéro). Leur représentation dans les modèles intégrés reste encore relativement partielle et constitue de nouvelles frontières dans les années à venir.

\section{Conclusion}

Longtemps parent pauvre des recherches consacrées à l'évaluation des politiques climatiques, l'analyse des cobénéfices des politiques climatiques a connu ces dernières années une indéniable montée en puissance, comme l'attestent les efforts de synthèse des évaluations dans le cinquième rapport du GIEC. Des programmes de recherche de premier plan comme le GEA ont redonné une visibilité à cette question, en particulier au sein de la communauté des modèles intégrés. Ces outils numériques permettent en effet d'évaluer les cobénéfices en représentant au travers de différents modules plus ou moins couplés les interactions entre les dimensions socioéconomiques, énergétiques et environnementales. L'évaluation des cobénéfices présente néanmoins encore de sérieux enjeux méthodologiques pour les modèles intégrés, en particulier dans le cadre de l'analyse coûtsbénéfices, à la fois pour représenter l'ensemble des cobénéfices et évaluer leurs impacts en matière de bienêtre pour la société. Les nouvelles dynamiques au sein des négociations climatiques caractérisées par une plus grande prise en compte des enjeux de développement sous la pression des pays du Sud tendent également à modifier le regard porté par les modèles intégrés sur les cobénéfices. Le climat n'est plus en tête des priorités, mais un objectif parmi d'autres qui s'intègre dans des stratégies nationales de développement spécifiques à chaque pays. Dans cette perspective, les évaluations sont amenées à s'intéresser davantage aux cobénéfices des politiques non climatiques dont le rôle dans les émissions de GES est significatif. La représentation dans les modèles intégrés $\mathrm{d}^{\prime}$ une gamme plus large de leviers d'action en matière de réduction des émissions, afin de prendre en compte les enjeux non climatiques, constitue une nouvelle frontière de recherche dans ce domaine. C'est sans doute en partie grâce à ces efforts méthodologiques, nécessaires pour évaluer les contributions nationales en matière de politiques climatiques qui structurent le processus des négociations actuelles en cours, qu'un dialogue, espérons-le constructif, peut s'établir entre modélisateurs et décideurs ${ }^{25}$.

\footnotetext{
${ }^{25}$ La place des évaluations modélisées dans l'élaboration des politiques publiques est encore difficile à cerner, même si le développement de méthodes d'aide à la décision multicritères, afin de mieux intégrer dans l'élaboration des politiques publiques les cobénéfices (Dubash et al., 2013), est sans doute une occasion d'utiliser davantage ces modèles comme outils d'aide à la décision. À noter par exemple la mise au point par l'IIASA d'une méthode d'aide à la décision dans le cadre du GEA qui a recours à l'utilisation du modèle intégré MESSAGE (http:/ / www.iiasa.ac.at/web/home/research/researchPrograms/Energy/User-Manual_ENE-MCA_2013-05-27.pdf).
} 


\section{Remerciements}

Les auteurs remercient le relecteur anonyme de l'article et l'éditeur pour ses commentaires utiles et éclairants.

\section{Références}

Ambrosi, P., Hourcade, J.-C., Hallegatte, S., Lecocq, F., Dumas, P., Ha-Duong, M., 2003. Optimal control models and elicitation of attitudes towards climate damages, Environmental Modeling and Assessment, 8, 3, 133-147.

Anderson, S.T., Parry, I.W., James, H., Sallee, M., Fischer, C., 2011. Automobile Fuel Economy Standards: Impacts, Efficiency, and Alternatives, Review of Environmental Economic and Policy, 5, 1, 89-108.

Aykut, S., Dahan, A., 2015. Gouverner le Climat? Vingt ans de négociations internationales, Paris, Presses de Sciences Po.

Barker, T., Johnstone, N., O'Shea, T., 1993. The CEC Carbon/ Energy Tax and Secondary Transport Related Benefits. EnergyEnvironment-Economy Modelling Discussion Paper No. 5, University of Cambridge, Cambridge.

Bodansky, D., Diringer, E., 2014. Evolution of the International Climate Effort, Arlington, Center for Climate and Energy Solutions.

Bussolo, M., O'Connor, D., 2001. Clearing the Air in India: The Economics of Climate Policy with Ancillary Benefits. Working Paper No. 182, OCDE, Paris.

Clarke, L., Jiang, K. Akimoto, K., Babiker, M., Blanford, G., Fisher-Vanden, K., Hourcade, J.-C., Krey, V., Kriegler, E., Löschel, A., McCollum, D., Paltsev, S., Rose, S., Shukla, P. R., Tavoni, M., van der Zwaan, B.C.C., van Vuuren, D.P., 2014. Assessing Transformation Pathways, in Climate Change 2014 Mitigation of Climate Change: Contribution of Working Group III to the Fifth Assessment Report of the Intergovernmental Panel on Climate Change [Edenhofer, O., Pichs-Madruga, R. Sokona, Y. Farahani, E. Kadner, S. Seyboth, K., Adler, A., Baum, I., Brunner, S., Eickemeier, P., Kriemann, B., Savolainen, J., Schlömer, S., von Stechow, C., Zwickel, T., Minx, J.C. (Eds)], Cambridge (UK), New York, Cambridge University Press.

Combet, E., 2013. Fiscalité carbone et progrès social : application au cas français. Thèse de doctorat, EHESS, Paris.

Complainville, C., Martins, J., 1994. NOx/SOx Emissions and Carbon Abatement. Working Papers No.151, OECD Economics Department, OECD Publishing (online: http:/ / dx.doi.org/10.1787/345333447174).

Damian, M., 2014. La politique climatique change enfin de paradigme, Économie Appliquée, 17, 1, 37-72.

Dubash, N.K., 2013. The politics of climate change in India: Narratives of equity and cobenefits, Wiley Interdisciplinary Review of Climate Change, 4, 3, 191-201.

Dubash, N.K., Raghunandan, D., Sant, G., Sreenivas, A., 2013. Indian climate change policy: exploring a co-benefits based approach, Economic and Political Weekly, 48, 22, 47-61.

Garbaccio, R.F., Ho, M.S., Jorgenson, D.W., 1999. Controlling carbon emissions in China, Environment and Development Economics, 4, 4, 493-518.
Glomsrød, S., Vennemo, H., Johnsen, T., 1992. Stabilization of emissions of $\mathrm{CO}_{2}$ : A computable general equilibrium assessment, Scandinavian Journal of Economics, 94, 1, 53-69.

Goulder, L.H., 1995. Environmental taxation and the double dividend: A reader's guide, International Tax and Public Finance, 2, 157-183.

Grubb, M., Vrolijk, C., Brack, D., 1999. The Kyoto Protocol: A guide and Assessment, London, RIIA/Earthscan, Washington, Brookings.

Gupta, J., 2014. The History of Global Climate Governance, Cambridge (UK), Cambridge University Press.

Hourcade, J.-C., Shukla, P.R., Mathy, S., 2008. Untying the climate - Development Gordian knot: Economic options in a politically constrained world, in Guesnerie, R., Tulkens, H., The Design of Climate Policy, Cambridge (MA), MIT Press.

Hourcade, J.-C., Shukla, P.R., 2013. Triggering the low-carbon transition in the aftermath of the global financial crisis, Climate Policy, 13, Supplement 1: Low carbon drivers for a sustainable world, 22-35.

IIASA, 2012. Global Energy Assessment (GEA): Toward a Sustainable Future, Cambridge (UK), Cambridge University Press.

IPCC, 1996. Mitigation of Climate Change: Contribution of Working Group III to the Fourth Assessment Report of the Intergovernmental Panel on Climate Change, Cambridge (UK), New York, Cambridge University Press.

IPCC, 2007. Mitigation of Climate Change: Contribution of Working Group III to the Fourth Assessment Report of the Intergovernmental Panel on Climate Change, New York, Cambridge University Press.

IPCC, 2013. The Physical Science Basis: Contribution of Working Group I to the Fifth Assessment Report of the Intergovernmental Panel on Climate Change, New York, Cambridge University Press.

IPCC, 2014. Climate Change 2014: Mitigation of Climate Change: Contribution of Working Group III to the Fifth Assessment Report of the Intergovernmental Panel on Climate Change [Edenhofer, O., Pichs-Madruga, R. Sokona, Y. Farahani, E. Kadner, S. Seyboth, K., Adler, A., Baum, I., Brunner, S., Eickemeier, P., Kriemann, B., Savolainen, J., Schlömer, S., von Stechow, C., Zwickel, T., Minx, J.C. (Eds)], Cambridge (UK) and New York, Cambridge University Press.

Krupnick, A., Burtraw, D., Markandya, A., 2000. The ancillary benefits and costs of climate change mitigation: A conceptual framework, in OECD (Ed.), Ancillary Benefits and Costs of Greenhouse Gas Mitigation, Paris, 53-93.

Lecocq, F., 2015. L'évaluation des politiques climatiques à l'échelle globale et nationale, in Torquebiau, E. (Ed.), Changer d'habitudes : changement climatique et agriculture du monde, Versailles, Quae, 279-291.

Markandya, A., Rübbelke, D.T.G., 2004. Ancillary benefits of climate policy, Journal of Economics and Statistics, 224, 4, 488503.

Markandya, A., Armstrong, B.-G., Hales, S., Chiabai, A., Criqui, P., Mima, S., Tonne, C., Wilkinson, P., 2009. Public health benefits of strategies to reduce greenhouse-gas emissions: Low-carbon electricity generation, The Lancet, 374, 9706, 2006-2015. 
McCollum, D.L., Krey, V., Riahi, K., 2011. An integrated approach to energy sustainability, Nature Climate Change, 1, 9, 428-29.

McCollum, D.L., Krey,V., Riahi, K., Kolp, P., Grubler, A., 2013. Climate policies can help resolve energy security and air pollution challenges, Climatic Change, 119, 2, 479-94.

OECD, 2000. Ancillary Benefits and Costs of Greenhouse Gas Mitigation, Paris, OECD.

O'Connor, D., Zhai, F., Aunan, K., Berntsen, T., Vennemo, H., 2003. Agricultural and Human Health Impacts of Climate Policy in China: A General Equilibrium Analysis with Special Reference to Guandong. Responding to Local and Global Environmental Challenges, Paris, OECD.

Pearce, D.W., 1976. Limits of cost-benefit analysis as a guide to environmental policy, Kyklos, 29, 1, 97-112.

Pearce, D.W., 1992. Secondary benefits of greenhouse gas control. CSERGE Working Paper, No 92-12, University of East Anglia and University College London, London.

Pearce, D.W., 2000. Policy framework for the ancillary benefits of climate change policies, in OECD (Ed.), Ancillary Benefits and Costs of Greenhouse Gas Mitigation, Paris, 517-560.

Pearce, D.W., 2001. Controversies in environmental valuation, in McMahon, P., Moran, D. (Ed.), Economic Valuation of Water Resources: Policy and Practice, London, Terence Dalton, 49-63.

Riahi, K., Dentener, F., Gielen, D., Grubler, A., Jewell, J., 2012. Chapter 17: Energy pathways for sustainable development, in Global Energy Assessment: Toward a Sustainable Future, Cambridge (UK), Cambridge University Press, 1203-1306.

Sarofim, M., Reilly, J., 2011. Applications of integrated assessment modeling to climate change, Wiley Interdisciplinary Review: Climate Change, 2, 1, 27-44.

Shukla, P.R., Dhar, S., 2011. Climate agreements and India: Aligning options and opportunities on a new track, International Environmental Agreements: Politics, Law and Economics, 11, 3, 229-243.

Sims, R., Schaeffer, R., Creutzig, F., Cruz-Núñez, X., D’Agosto, M., Dimitriu, D., Figueroa Meza, M.J., Fulton, L., Kobayashi, S., Lah, O., McKinnon, A., Newman, P., Ouyang, M., Schauer, J.J., Sperling, D., Tiwari, G., 2014.
Transport, in Climate Change 2014: Mitigation of Climate Change: Contribution of Working Group III to the Fifth Assessment Report of the Intergovernmental Panel on Climate Change [Edenhofer, O., Pichs-Madruga, R. Sokona, Y. Farahani, E. Kadner, S. Seyboth, K., Adler, A., Baum, I., Brunner, S., Eickemeier, P., Kriemann, B., Savolainen, J., Schlömer, S., von Stechow, C., Zwickel, T., Minx, J.C. (Eds)], Cambridge University Press, Cambridge (UK) and New York.

Sovacool, B.K., Brown, M.A., 2010. Competing dimensions of energy security: An international perspective, Annual Review of Environment and Resources, 35, 77-108.

The White House, 2011. Blueprint for a Secure Energy Future. Washington D.C. (online: http://www.whitehouse.gov/ sites/default/files/blueprint_secure_energy_future.pdf).

UNFCCC, 2014. Lima Call for Climate Action. Decision 1/ CP.20 (online: http://unfccc.int/resource/docs/2014/ cop20/eng/10a01.pdf).

Ürge-Vorsatz, D., Tirado Herrero, S., Dubash, N.K., Lecocq, F., 2014. Measuring the co-benefits of climate change mitigation, Annual Review of Environment and Resources, 39, 1, 549-582.

Waisman, H., Guivarch, C., Grazi, F., Hourcade, J.-C. 2012. The Imaclim-R model: Infrastructures, technical inertia and the costs of low carbon futures under imperfect foresight, Climatic Change, 114, 1, 101-120.

Waisman, H., Rozenberg, J., Hourcade, J.-C., 2013. Monetary compensations in climate policy through the lens of a general equilibrium assessment: The case of oil-exporting countries, Energy Policy, 63, 951-961.

West, J.J., Smith, S.J., Silva, R.A., Naik, V., Zhang, Y., Adelman, Z., Fry, M., Anenberg, S., Horowitz, L.W., Lamarque, J.-F., 2013. Co-benefits of mitigating global greenhouse gas emissions for future air quality and human health, Nature Climate Change, 3, 885-889.

World Bank, 2014. Turn Down the Heat: Confronting the New Climate Normal. World Bank, Washington D.C. (online: https://openknowledge.worldbank.org/handle/10986/ 20595). 